\title{
Septic thrombophlebitis of the internal jugular vein, a case of Lemierre's syndrome
}

\author{
Adam Alperstein ${ }^{1}$, Raymond M. Fertig ${ }^{2, *}$, Matthew Feldman², Daniel Watford ${ }^{2}$, Susan Nystrom ${ }^{1}$, \\ Guesly Delva ${ }^{1}$, Salman Muddassir ${ }^{1}$
}

${ }^{1}$ Oak Hill Hospital, Graduate Medical Education, Department of Internal Medicine, Brooksville, FL, USA,

${ }^{2}$ University of Miami, Miller School of Medicine, Miami, FL, USA.

\begin{abstract}
Summary An 18-year-old gentleman with a history of recurrent tonsillitis presented to the emergency room complaining of worsening sore throat. He was found to have a peritonisillar abscess, and imaging revealed a non-occlusive left internal jugular vein thrombosis. Lemierre's syndrome is a rare, potentially fatal condition characterized by internal jugular vein thrombosis with septicemia following an acute oropharyngeal infection. While anticoagulation is the mainstay of treatment of deep venous thromboembolism (DVT) and pulmonary embolism (PE), the use of therapy is controversial in septic thrombophlebitis. This is counterintuitive since a common reported complication is pulmonary emboli. Early in the course of thrombophlebitis, while the thrombus is firmly attached, antibiotics may be all that is necessary to treat the condition.
\end{abstract}

Keywords: Lemierre's syndrome, Fusobacterium necrophorum, postanginal septicemia, septic thrombophlebitis

\section{Introduction}

Lemierre's syndrome is a rare, potentially fatal condition characterized by internal jugular vein (IJV) thrombosis with septicemia following an acute oropharyngeal infection. Lemierre's syndrome (LS) has been termed the "forgotten disease" due to the unfamiliarity of many physicians with this syndrome because of its rarity, with less than one case per million occurring in the general population (1). LS is typically associated with a gram-negative retropharyngeal abscesses leading to septic metastasis to the vasculature of the head and neck (2). The most commonly associated organism is Fusobacterium necrophorum, a gramnegative, anaerobic rod-shaped bacterium that is part of the normal oral flora (3). Other associated bacterium include Streptococcal species such as Streptococcus

Released online in J-STAGE as advance publication May 24, 2017.

*Address correspondence to:

Dr. Raymond Fertig, University of Miami, Miller School of Medicine, Department of Dermatology and Cutaneous Surgery, 1475 NW 12th Ave, 2nd Floor Miami Florida, 33136, USA.

E-mail: raymondfertig@gmail.com pyogenes (1), Eikenella corrodens (4), and Bacteroides species (5). The venous thrombosis associated with this syndrome is secondary to endothelial dysfunction caused by inflammatory factors from the local infection and can be treated with antibiotics alone, often without the need for anticoagulation therapy. We report a case of Lemierre's syndrome in an 18-year-old male adolescent who developed a left internal jugular vein thrombosis following an acute oropharyngeal infection. The patient was successfully managed with incision and drainage (I\&D) of the developing peritonsillar abscess in conjunction with intravenous (IV) antibiotics.

\section{Case Report}

An 18-year-old gentleman with a history of recurrent tonsillitis presented to the emergency room complaining of worsening sore throat. Three days prior he was diagnosed with pharyngitis in the outpatient setting where he was prescribed oral amoxicillin and clindamycin. However, his symptoms continued to progress, including dysphagia, odynophagia, change of voice, and difficulty managing secretions, though without concurrent dyspnea.

The patient had no known drug allergies and was not taking any other home medications. His past 
medical history was significant for recurrent tonsillitis, tonsilloliths, and a left sided peritonsillar abscess treated with I\&D approximately 5 years prior.

On physical exam, vital signs were temperature $98.7^{\circ} \mathrm{F}$, blood pressure $122 / 73 \mathrm{~mm} \mathrm{Hg}$, heart rate $111 \mathrm{bpm}$, respiratory rate $18 \mathrm{bpm}$, and $98 \%$ oxygen saturation on room air. He was in no acute distress without wheezing, stridor or signs of respiratory distress. There was left peritonsillar swelling, fullness and mild tenderness with right shift of the uvula. The hypopharynx and larynx could not be visualized. He also had mild tenderness near the left jaw angle that was exacerbated by turning his head to the left. The neck was nontender to palpation without lymphadenopathy. The remainder of the exam was otherwise benign.

Initial labs were significant for a leukocytosis of $16.6 \times 10^{3}$ cell $/ \mu \mathrm{L}$. A rapid strep and monospot test

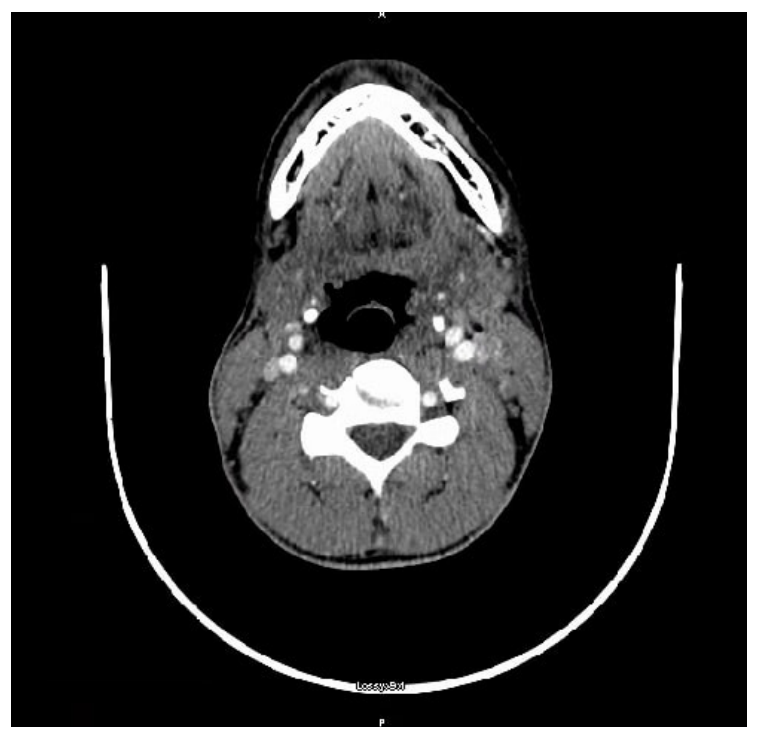

Figure 1. Computed tomography image of the neck: Left-sided peritonsillar phelgmon with associated reactive adenopathy. Note the left internal jugular vein, with an area of limited opacification, concerning for possible thrombosis. were both negative. A computed tomography (CT) scan of the neck revealed a left-sided peritonisillar phelgmon with a $9 \mathrm{~mm}$ fluid collection suspicious for a developing abscess. It also demonstrated a leftsided internal jugular vein abnormality, concerning for thrombosis (Figure 1). Doppler ultrasound of the neck confirmed a nonocclusive thrombus in the left internal jugular vein (IJV). (Figures 2A and 2B)

The patient was admitted with the diagnosis of sepsis secondary to left sided peritonsillar abscess complicated with left IJV thrombosis, with microorganism not yet determined. Cultures were drawn and he was started empirically on broad spectrum IV antibiotics with vancomycin and ampicillin/sulbactam. In addition, solumedrol was also administered and ENT was consulted. At that time the question arose whether to start the patient on anticoagulation therapy for the thrombus. The decision was made to hold off on anticoagulation therapy and re-evaluate the thrombus following surgery.

The patient was taken to the operating room for I\&D of the peritonsillar abscess and approximately $10 \mathrm{cc}$ of pus was aspirated. He was continued on broad spectrum IV antibiotics and reported immediate clinical improvement postoperatively. On hospital day three his leukocytosis was improving and a repeat ultrasound of the neck showed very minimal residual thrombosis of the left internal jugular vein. Gram stain of the aspirated fluid demonstrated 2+ WBCs, $2+$ gram positive cocci in pairs and chains, and $1+$ gram positive rods, with few mixed anaerobic flora. Although speciation and sensitivities were unavailable, the antibiotics were de-escalated to ceftriaxone and metronidazole. A peripherally inserted central venous catheter (PICC) line was placed for the patient to continue IV antibiotics in the outpatient setting in order to complete a 2-week course. He was advised to follow up with ENT for interval tonsillectomy in 4-6 weeks to prevent recurrence.

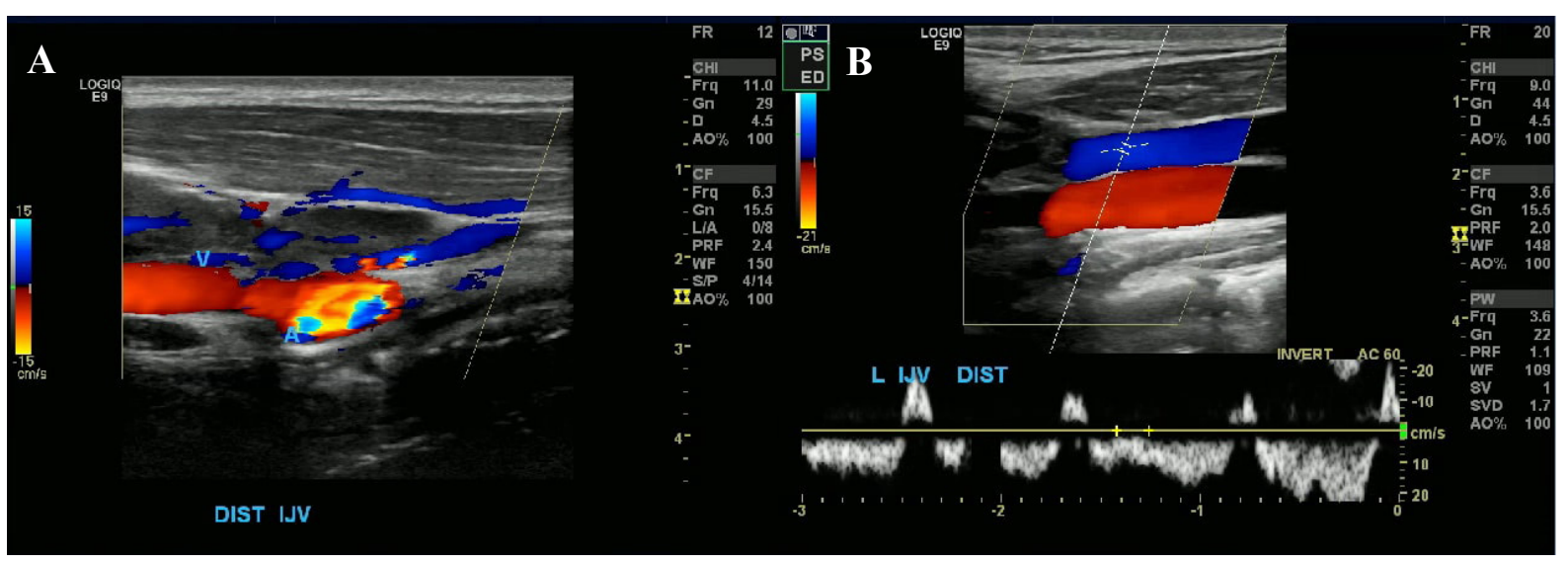

Figure 2. Ultrasound of the neck: (A), intraluminal echogenic filling defect within the distal left jugular vein which was noncompressible, consistent with a non-occlusive thrombus. (B), repeat ultrasound after the initiation of therapy demonstrating very minimal residual thrombosis of the distal IJV. 


\section{Discussion}

Suppurative thrombophlebitis of the internal jugular vein (IJV) is a venous thrombosis due to the infectious involvement of the carotid sheath vessels with bacteria and is seen in association with intravenous catheters or with certain deep neck infections (5). It was initially described in 1936 by Andre Lemierre as postanginal septicemia but has also been referred to as Lemierre's Syndrome. Lemierre's syndrome is described as a rare, potentially fatal condition characterized by internal jugular vein thrombosis with septicemia following an acute oropharyngeal infection. The infectious involvement of the carotid sheath vessels can arise from the normal oropharyngeal flora, most notably Fusobacterium necrophorum, but other organisms have also been implicated. It has a propensity to affect young healthy adults, with a mean age of 20 years, often occurring within one week of onset of pharyngitis (5). Incidence is between 0.6 and 2.3 per million with a mortality rate between $4 \%$ and $18 \%$ (6). Septic pulmonary emboli are a common sequelea, reported in as high as $97 \%$ of cases $(5,7,8)$. This may lead to hypoxemia and empyema among other potential consequences.

Lemierre's syndrome was once fatal, but the incidence has drastically decreased due to the wide spread use of antibiotics. This decrease has led to Lemierre's syndrome often being referred to as "the forgotten disease" due to its rarity and unfamiliarity among physicians. However, there may be a resurgence in Lemierre's related to recent restriction of antibiotic use for upper respiratory tract infections which are frequently presumed to be viral in etiology (9).

One should suspect IJV thrombophlebitis in a patient with pharyngitis whose symptoms do not resolve within 3-5 days, a rapidly worsening course, unilateral neck swelling, and systemic symptoms with persistent fever or bacteremia. Early recognition is vital to prevent sepsis and death, yet the diagnosis is often delayed because of the indolent course and obscurity of the syndrome. The diagnosis was previously made with blood cultures showing F. necrophorum. However, appropriate imaging findings utilizing CT can precede blood culture results (10). A CT scan of the neck with contrast is the most useful study to detect internal jugular vein thrombosis (7). Due to the frequency of pulmonary emboli that accompanies this condition, a CT scan of the chest is often performed. Furthermore, ultrasonography is also useful to evaluate for jugular vein thrombosis and can be used to assess for thrombus extension (7). In addition, microbiologic diagnosis may be performed with blood cultures or cultures from purulent material expressed from the site.

Prior to antibiotics, the treatment for Lemierre's was primarily surgical ligation and excision. Today, the treatment, similar to other forms of septic thrombophlebitis, involves removing the foci of infection, such as with catheter removal or incision and drainage of an abscess, prompt antibiotic administration, and a consideration for possible anticoagulation (7). Since beta lactamase production has been identified in some Fusobacterium species, empiric antibiotic selection should include a beta lactamase resistant beta lactam, such as ampicillin-sulbactam (11).

While anticoagulation is the mainstay of treatment of deep venous thromboembolism (DVT) and pulmonary embolism (PE), the use of therapy is controversial in septic thrombophlebitis. Despite the potential life threatening complications mentioned in the literature, the role of anticoagulation is not recommended in the absence of extension of the thrombus (12). In general, the mechanism of the circulatory disturbance in a particular thrombotic disease process should be understood prior to the initiation of anticoagulation therapy. We know that Virchow's triad describes that a thrombus formation can be due to venous stasis, a hypercoagulable state, and endothelial injury. The accepted mechanism of Lemierre's is that certain deep neck infections in predisposed individuals by a strain of bacteria producing endotoxins and hemagglutinin leading to further invasion, endothelial inflammation, platelet aggregation, and a suppurative thrombophlebitis. Early in the course of thrombophlebitis, while the thrombus is firmly attached, antibiotics may be all that is necessary to treat the condition.

There are various complex mechanisms leading to such thrombotic events. Perhaps further studies can elucidate the most appropriate management of these thrombotic events, possibly not requiring anticoagulation to improve clinical outcomes in patients. Clinicians should suspect jugular vein suppurative thrombophlebitis in patients with pharyngitis, septic pulmonary emboli, and persistent fever despite antimicrobial therapy.

\section{References}

1. Chamseddin KH, Kirkwood ML. Lemierre's syndrome associated mycotic aneurysm of the external carotid artery with primary internal carotid artery occlusion in a previously healthy 18-year-old female. Ann Vasc Surg. 2016; 36:291.e11-291.e14.

2. Karkos PD, Asrani S, Karkos CD, Leong SC, Theochari EG, Alexopoulou TD, Assimakopoulos AD. Lemierre's syndrome: A systematic review. The Laryngoscope. 2009; 119:1552-1559.

3. Celikel TH, Muthuswamy PP. Septic pulmonary emboli secondary to internal jugular vein phlebitis (postanginal sepsis) caused by Eikenella corrodens. Am Rev Respir Dis. 1984;130:510-513.

4. Sinave CP, Hardy GJ, Fardy PW. The Lemierre syndrome: Suppurative thrombophlebitis of the internal jugular vein secondary to oropharyngeal infection. Medicine. 1989; 68:85-94.

5. Vargiami EG, Zafeiriou DI. Eponym: The Lemierre syndrome. Eur J Pediatr. 2010; 169:411-414. 
6. Golpe R, Marín B, Alonso M. Lemierre's syndrome (necrobacillosis). Postgrad Med J. 1999; 75:141-144.

7. Kobayashi Y, Takayanagi N, Sugita Y. A case of Lemierre's syndrome with septic pulmonary embolisms. Kansenshogaku Zasshi. 2014; 88:695-699. (in Japanese)

8. Ramirez S, Hild TG, Rudolph CN, Sty JR, Kehl SC, Havens P, Henrickson K, Chusid MJ. Increased diagnosis of Lemierre syndrome and other Fusobacterium necrophorum infections at a Children's Hospital. Pediatrics. 2003; 112:e380.

9. Screaton NJ, Ravenel JG, Lehner PJ, Heitzman ER, Flower CD. Lemierre syndrome: Forgotten but not extinct - report of four cases. Radiology. 1999; 213:369-374.

10. Lin D, Reeck JB, Murr AH. Internal jugular vein thrombosis and deep neck infection from intravenous drug use: Management strategy. Laryngoscope. 2004; 114:5660 .

11. Prandoni P, Tormene D, Pesavento R; Vesalio Investigators Group. High vs. low doses of low-molecular-weight heparin for the treatment of superficial vein thrombosis of the legs: A double-blind, randomized trial. J Thromb Haemost. 2005; 3:1152-1157.

12. Phua CK, Chadachan VM, Acharya R. Lemierre syndrome - should we anticoagulate? A case report and review of the literature. Int J Angiol. 2013; 22:137-142.

(Received April 8, 2017; Revised May 16, 2017; Accepted May 18, 2017) 